\title{
Evidence of Pneumocystis carinii in cell line cultures infected with peripheral blood mononuclear cells isolated from AIDS patients with $P$. carinii pneumonia
}

\author{
C. CONTINI, S. MASTRANTONI*, R. ROMANI $\dagger$, R. CULTRERA and S. DELIA $\dagger$
}

Institute of Infectious and Respiratory Diseases, University of Ferrara, 44100 Ferrara and Departments of * Tropical and †Infectious Diseases, "La Sapienza" University, Rome, Italy

\begin{abstract}
Summary. The detection of Pneumocystis carinii was investigated in an in-vitro system consisting of a human lung epithelial cell line (A-549) inoculated with infected peripheral blood mononuclear cells (PMBC) from HIV-infected patients with proven or suspected $P$. carinii pneumonia (PCP), and from HIV-negative patients with other lung infections. Supernates from cultures were sampled daily and evaluated for the presence of $P$. carinii by Giemsa and immunofluorescence staining. P. carinii was isolated from $98(95 \cdot 1 \%)$ of 103 culture supernate samples from patients with proven pneumocystosis and $45(66.1 \%)$ of 68 from patients with suspected PCP 40 or $72 \mathrm{~h}$ after PBMC inoculation. This system has been shown to support the growth of $P$. carinii but did not seem to be adequate for the production of large numbers of organisms, although long-term survival in vitro for up to 3 weeks was observed. Recovery of $P$. carinii from infected PBMC strongly supports previous observations about its ability to disseminate haematogenously and could represent a further advance in understanding the pathogenesis and diagnosis of PCP.
\end{abstract}

\section{Introduction}

Pneumocystis carinii causes lethal pneumonia (PCP) in immunosuppressed patients and is a leading cause of mortality in patients with acquired immunodeficiency syndrome (AIDS). Pneumocystosis is diagnosed currently by direct visualisation of the organism in infected lung tissue or in respiratory fluids by conventional cytochemical or immunocytochemical staining. Immunological assays have also been employed. However, these methods are of little indicative value in $P$. carinii disease and current opinion is that the detection of antibodies or circulating soluble antigen cannot be considered to be alternatives to invasive diagnostic procedures.

In the last few years, new techniques have been developed for the diagnosis of PCP. In particular, detection of $P$. carinii in blood by the polymerase chain reaction (PCR) has provided a highly sensitive and specific tool that has facilitated diagnosis and elucidated the natural history and epidemiology of pneumocystosis. ${ }^{1-4}$

Considering the potential diagnostic value of the detection of the organism in the blood and because approaches to this problem have never involved invitro procedures, a modified culture system consisting of a human lung epithelial cell line (A-549) with inocula derived from peripheral blood mononuclear cells (PBMC) from AIDS patients with PCP was employed to determine whether it could be useful in detecting $P$. carinii infection.

Accumulating evidence suggests that attachment to host cells, particularly alveolar epithelial cells, is an important step for the growth and replication of the parasite and appears to be mediated by cell-surface glycoproteins, exogenous fibronectin and components of the parasite cytoskeleton. ${ }^{5}$ At no time in its life cycle does $P$. carinii seem to be an intracellular pathogen.

A protective host response against $P$. carinii involves both functional CD4 $+\mathrm{T}$ lymphocytes and alveolar macrophages (AM). Studies in vitro have shown that the organism can be ingested and digested by normal $\mathrm{AM}$ and killed by macrophage-derived biochemical mediators, including cytokines, which may contribute to the control of pneumonia. ${ }^{6}$ In-vitro studies have also demonstrated that conditions that lead to defects in the host's immune response, such as AIDS or corticosteroid treatment, may impair the phagocytic and cytolytic activities of $\mathrm{AM}$ by altering membrane $\mathrm{Fc}$ receptor functions. 
The detection of $P$. carinii in cultured PBMC could be useful for the diagnosis of PCP, and also for providing insights into the relationship between this organism and circulating human monocytes in the pathobiology of this infection.

\section{Materials and methods}

\section{Clinical specimens and PBMC isolation}

Single or multiple blood samples were obtained as part of routine viral surveillance from 64 outpatients attending the AIDS clinic or patients admitted to the Institute of Infectious Diseases of University "La Sapienza", Rome, between September 1991 and February 1993. The average age was 34.7 years (range 28-51 years) for male patients and 31.2 years (range 24-44 years) for female patients. Thirty-eight were AIDS patients with morphologically and clinically proven PCP or extrapulmonary pneumocystosis (one case); 17 were patients who presented with respiratory symptoms, severe lung involvement and gasometric features consistent with PCP, in whom neither bronchoalveolar lavage (BAL) nor induced sputum could be obtained to make a definitive laboratory diagnosis (these subjects were not known to be on prophylaxis or treatment for PCP and no other pathogen was isolated from their samples by routine examination); nine were AIDS patients who were febrile and had other causes of pneumonias (Mycobacterium tuberculosis, four patients; Streptococcus pneumoniae, three; cytomegalovirus, two. Forty healthy afebrile volunteers without respiratory diseases were also included in the study.

PBMC were isolated from $10 \mathrm{ml}$ of heparinised whole blood by Ficoll Hypaque density gradient centrifugation (Lymphoprep, Nycomed, Oslo, Norway) in plastic $50-\mathrm{ml}$ centrifugation tubes at $400 \mathrm{~g}$ at room temperature for $30 \mathrm{~min}$, within $2 \mathrm{~h}$ after collection. The top band at the sample-medium interface consisted of mononuclear cells and the lower band of polymorphonuclear cells. The band of mononuclear cells was washed twice with $10 \mathrm{ml}$ of phosphatebuffered saline and centrifuged at $200 \mathrm{~g}$ for $8 \mathrm{~min}$.

\section{A-549 cell culture}

PBMC from each blood sample were then inoculated on to 48-h confluent A-549 cell monolayers grown in triplicate $25-\mathrm{cm}^{2}$ flasks (ICN, Costa Mesa, CA, USA) in Ham's F12 medium (Slavo, Siena, Italy) $90 \%$, supplemented with fetal calf serum $10 \%$, antibiotics (ampicillin 200 U/ml, streptomycin $200 \mu \mathrm{g} / \mathrm{ml}$, amphotericin B $0.5 \mu \mathrm{g} / \mathrm{ml}$ ) and incubated at $37^{\circ} \mathrm{C}$ in a humidified $\mathrm{CO}_{2} 5 \%$ atmosphere. After inoculation, cultures were sampled daily by removing one-tenth of the volume of each flask and pooling supernates as reported previously.?

The pooled supernates from ongoing cultures were centrifuged at $5000 \mathrm{~g}$ and then checked for $P$. carinii nuclei and cyst counts by a modified Giemsa stain ${ }^{8}$ and immunofluorescence staining with monoclonal anti- bodies (MAbs) to human strains of $P$. carinii. $^{9}$ The viability of the micro-organisms was determined by a combination stain with fluorescein diacetate and ethidium bromide ${ }^{10}$ adapted for $P$. carinii. To optimise the quantitation system, the number of $P$. carinii was calculated from each supernate sample by determining the mean number of cysts and trophozoites grown in triplicate flasks during the 3-week culture period. Statistical differences were calculated by analysis of variance.

\section{Controls for A-549 cell cultures}

To determine the conditions under which growth of human PBMC-derived $P$. carinii could be observed in vitro, a number of uninfected cultures were also inoculated with $P$. carinii extracts (inoculum size $=$ $1.5 \times 10^{6}$ cysts) from frozen stored post-mortem human lung tissue prepared according to the method of Walzer et al., ${ }^{11}$ and with non-infected human lung treated in the same manner as the $P$. carinii-infected tissue. For $P$. carinii inocula as well as for normal lung tissues, erythrocytes were lysed with ammonium chloride $0.85 \%$. Cultures were monitored for the presence of bacteria and fungi by microscopy and culture. ${ }^{12}$

\section{Results}

In separate experiments, two non-commercial wellcharacterised MAbs were evaluated to establish their sensitivity and specificity. P. carinii cysts were identified clearly by indirect immunofluorescence in respiratory secretions as well as in cultured PBMC from patients with PCP. Specificity studies were done on each of the two MAbs with normal human lung extract and fungi such as Cryptococcus neoformans and various Candida spp., including C. albicans. No nonspecific fluorescence was observed. These MAbs have also been shown to be as sensitive and specific as a commercial Monofluo kit (Diagnostic Pasteur, France).$^{9} P$. carinii trophozoites were counted by microscopy with a modified Giemsa stain, which although less rapid than Diff Quik, allowed the assessment of organism growth without observation of background material. ${ }^{7}$

When an A-549 cell culture was infected with human lung-derived $P$. carinii, counts of both cyst and trophozoite forms peaked from day 1 to day 3 from the beginning of the culture. Cyst and trophozoite counts decreased from day 3 but cysts predominated in large clumps and persisted at levels below the inoculum size for up to 1 week of culture. These data indicated that an inoculum size of $1.5 \times 10^{6}$ cysts could be used to evaluate $P$. carinii growth in culture and to ensure uniformity of results.

In the cell cultures infected with human PBMC, cultures provided evidence of infection in $36(94.7 \%)$ of the 38 AIDS patients with proven pneumocystosis and in nine $(52.9 \%)$ of the 17 patients with suspected 


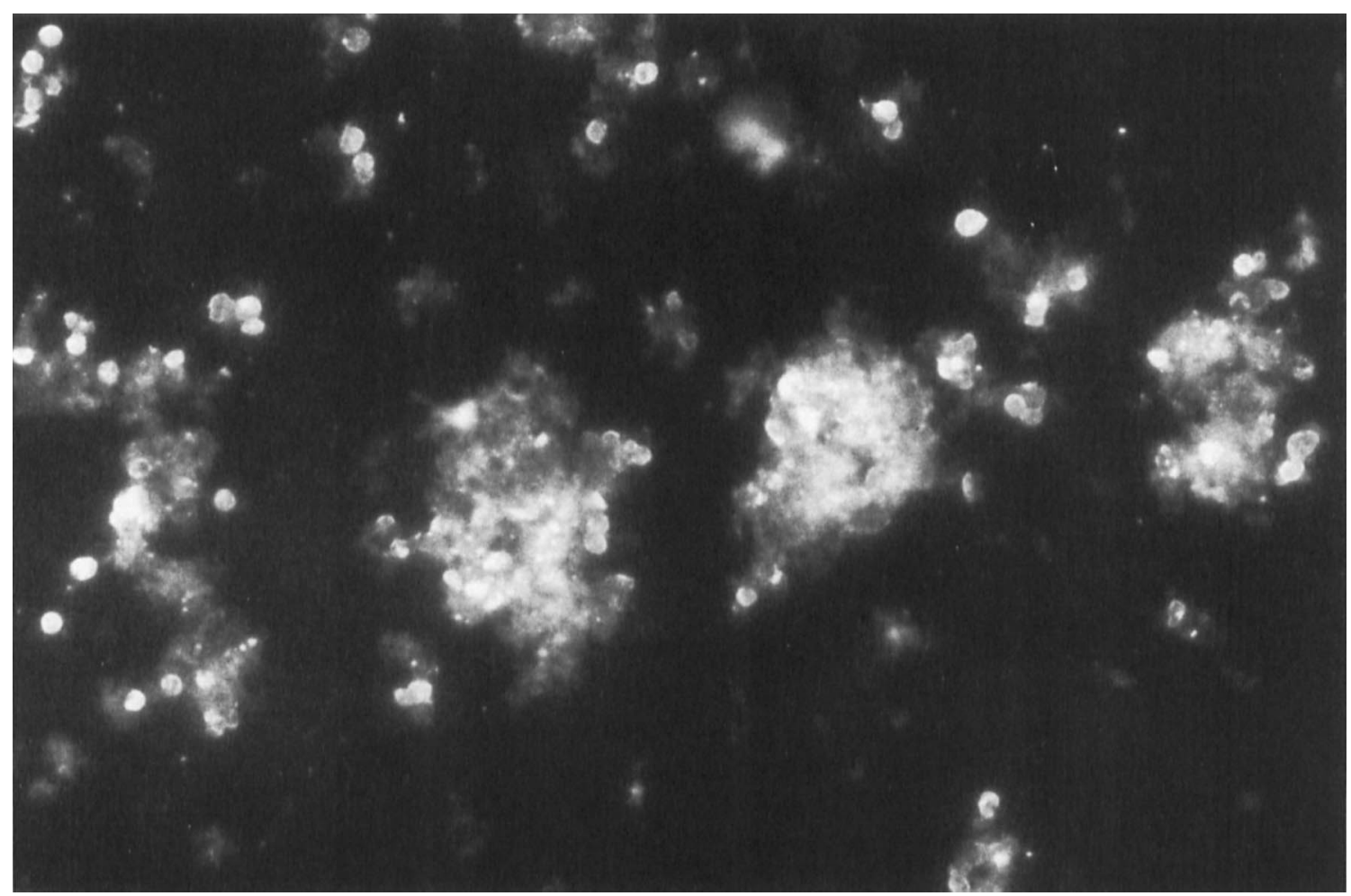

Figure. Immunofluorescence stain showing clusters of $P$. carinii organisms in a 3-day culture supernate of cell line A-549 inoculated with PBMC from a patient with PCP $(\times 400)$.

Table. Numbers of $P$. carinii organisms grown in cultured PBMC from different population groups

\begin{tabular}{|c|c|c|c|c|c|}
\hline \multirow{2}{*}{ Patient characteristic } & \multirow{2}{*}{$\begin{array}{l}\text { Number }(\%) \text { of } \\
\text { positive patients }\end{array}$} & \multirow{2}{*}{$\begin{array}{l}\text { Number }(\%) \text { of } \\
\text { positive cultures }\end{array}$} & \multicolumn{3}{|c|}{ Mean (SE) organism yield during 3 weeks of culture } \\
\hline & & & 1-7 days & $8-14$ days* & $15-21$ days $\dagger$ \\
\hline $\begin{array}{l}\text { AIDS, clinically and } \\
\text { microbiologically proven PCP } \\
(38)\end{array}$ & $36(94 \cdot 7)$ & $98 / 103(95 \cdot 1)$ & $2 \cdot 16(1 \cdot 15) \times 10^{5}$ & $1.64(0.2) \times 10^{4}$ & $1 \cdot 22(2 \cdot 0) \times 10^{3}$ \\
\hline $\begin{array}{l}\text { AIDS, clinically suspected PCP } \\
\text { (17) }\end{array}$ & $9(52 \cdot 9)$ & $45 / 68(66 \cdot 1)$ & $4.45(5.65) \times 10^{4}$ & $9.01(2.6) \times 10^{3}$ & $19 \cdot 89(5 \cdot 03)$ \\
\hline $\begin{array}{l}\text { AIDS, febrile, with other } \\
\text { pneumonias }(9)\end{array}$ & $0-$ & $0 / 42-$ & - & - & - \\
\hline Control subjects $(40)$ & $0-$ & $0 / 58-$ & - & - & - \\
\hline
\end{tabular}

Note: point data are expressed as the means of $P$. carinii forms from pooled samples of triplicate flasks.

${ }^{*}$ Organism counts significantly different between first and second week, $\mathrm{p}<0.01$. †Organism counts significantly different between second and third week, $\mathrm{p}<0.001$.

PCP. Culture supernate samples were positive $2-3$ days after PBMC inoculation, with large clusters of organisms which were predominantly the cyst form (figure). Different growth patterns were observed in supernate samples collected after the first week of the culture. Generally, trophozoites appeared to predominate and occurred scattered in groups. Although many of the organisms remained adherent to the underlying cells and could not be counted accurately, the number of these decreased significantly between the first and the second week $(p<0.01)$, as well as between the second and the third week $(\mathrm{p}<0.001)$ after inoculation (table). All positive cultures showed prolonged $P$. carinii survival for up to 3 weeks, after which most of the organisms were non-viable.

No evidence of $P$. carinii growth was observed on monolayers or in supernates inoculated with PBMC from patients with other respiratory infections (nonPCP) or from control subjects, nor from cultures inoculated with normal human lung extract. The negative culture specimens underwent no further testing. No fungal or bacterial contaminants were observed.

\section{Discussion}

In the last 10 years, different immunological approaches have been used for the diagnosis of pneumocystosis. Antibody testing has been shown to be useful epidemiologically, but it is unreliable in diagnosing PCP, as the majority of healthy individuals as well as patients with $P$. carinii infection have substantial titres. ${ }^{13}$ Antigen detection, although initially encour- 
aging, was also reported to be a poor diagnostic system because of the tight adherence of this pathogen to the host pneumocytes which could hinder its access to the bloodstream and subsequent release of antigen. ${ }^{14}$

With increasing reports of cases of disseminated pneumocystosis, which support the concept that organisms may reach and colonise disparate sites outside the lung, ${ }^{15,16}$ evidence is now emerging that $P$. carinii is liberated from the lung into the peripheral circulation through haematogenous or lymphatic pathways. Recently, $P$. carinii has been detected by PCR DNA amplification of blood from rats and human patients with PCP and with disseminated pneumocystosis, thus helping to answer a number of important questions about the pathogenesis of $P$. carinii infection. ${ }^{2,3}$

Considering the potential importance of detection of the organism in the blood, a culture method was used which has been employed extensively for the short-term culture of rat- or human-derived P. carinii. ${ }^{17.18}$ In the current study, the cell line was infected with a different inoculum source, which consisted of PBMC from human patients with $P$. carinii infection instead of conventional inocula.

This system was able not only to confirm cases of known pneumocystosis, but also to recognise the typical organisms in high isolation rates in culture supernate samples from AIDS patients with suspected PCP in whom demonstrations of $P$. carinii could not be made conventionally and in whom specific therapy for pneumocystosis was followed by rapid clinical and radiographic improvement. $P$. carinii forms were not seen in cultures infected with PBMC from HIVinfected patients with other pneumonias or from control subjects, thus confirming previous reports which did not reveal positive PCR signals in patients with advanced AIDS without a history of PCP. ${ }^{3}$

Having demonstrated the presence of $P$. carinii in cultured human-derived PBMC, we were not able to determine whether organisms were attached to these cells or were within the PBMC. The interaction of these organisms with monocytes and $\mathrm{T}$ cells was reported previously. ${ }^{19}$ Transmission electronmicroscopy has shown that $P$. carinii trophozoites adhere to alveolar and peritoneal macrophages and $\mathrm{T}$ cells, and

\section{References}

1. Lipschik GY, Gill VJ, Lundgren JD et al. Improved diagnosis of Pneumocystis carinii infection by polymerase chain reaction on induced sputum and blood. Lancet $1992 ; 340$ : 203-206.

2. Schluger N, Godwin T, Sepkowitz K et al. Application of DNA amplification to pneumocystosis; presence of serum Pneumocystis carinii DNA during human and experimentally induced Pneumocystis carinii pneumonia. J Exp Med 1992; 176: 1327-1333.

3. Sepkowitz K, Schluger N, Godwin T, Armstrong D, Cerami A, Bucala R. DNA amplification in experimental pneumocystosis: characterization of serum Pneumocystis carinii DNA and potential $P$. carinii carrier states. $J$ Infect Dis 1993; 168: $421-426$.

4. Wakefield AE, Pixley FJ, Banerji S et al. Detection of Pneumocystis carinii with DNA amplification. Lancet $1990 ; 336$ : $451-453$. are ingested and destroyed by macrophages after the addition of rabbit anti- $P$. carinii serum, thus suggesting that humoral and cellular mechanisms may be necessary in combination to control $P$. carinii disease. Some reports have also suggested that proteins such as fibronectin play an important role in attachment by serving as a bridge between the surface glycoprotein- $\mathrm{A}$ and host cell receptors. ${ }^{20}$ Recently, $P$. carinii surface glycoprotein-A was demonstrated to be a chemotactic factor for normal human blood monocytes. ${ }^{21}$

Presently, most of the cell-culture systems employed for $P$. carinii propagation have yielded only transient proliferation and did not prove adequate for isolation of $P$. carinii in sufficient quantities to explore important basic biological questions. Much of this unsuccessful work is related to the quality of the inocula used, which may be contaminated or not of sufficient purity. ${ }^{7,15,22,23}$ A culture system employing cell monolayers on micro-carrier beads has been described recently. This method did not achieve $P$. carinii proliferation significantly higher than that seen in plate or flask culture, but the organisms harvested contained little host-cell contamination. ${ }^{24}$

The system employed in the present report appears to support the growth of $P$. carinii and to avoid the technical problems resulting from contamination of host cells or additional infectious agents introduced with a conventional $P$. carinii inoculum. This method has been shown to be inadequate for the production of large numbers of organisms, even if cultured for 21 days. Humoral factors secreted by PBMC which allow $P$. carinii to grow in culture, or HIV infection of the monolayer cells by the PBMC somehow making the monolayer cells permissive to the organism's growth, might explain long term survival.

The possibility of identification of $P$. carinii in cultures infected with blood from patients with proven or suspected PCP represents a further advance in $P$. carinii diagnosis and better understanding of the transmission of the infection. A more extensive development of this in-vitro cultivation method could also allow a study of the putative differences between $P$. carinii strains isolated from the blood of infected individuals. ${ }^{25}$

5. Limper AH. Parasitic adherence and host responses in the development of Pneumocystis carinii pneumonia. Semin Respir Infect 1991; 6: 19-26.

6. Pesanti EL. Interaction of cytokines and alveolar cells with Pneumocystis carinii in vitro. J Infect Dis 1991; 163: $611-613$.

7. Cushion MT, Walzer PD. Growth and serial passage of Pneumocystis carinii in the A549 cell line. Infect Immun $1984 ; 44: 245-251$.

8. Walker J, Conner G, Ho J, Hunt C, Pickering L. Giemsa staining for cysts and trophozoites of Pneumocystis carinii. $J$ Clin Pathol 1989; 42: 432-434.

9. Nato F, Contini C, Zamora Zavala $C$ et al. Production and characterization of monoclonal antibodies to human Pneumocystis carinii for the diagnosis of $P$. carinii pneumonia. Eur J Med 1992; 1: 132-138.

10. Jackson PR, Pappas MG, Hansen BD. Fluorogenic substrate detection of viable intracellular and extracellular pathogenic protozoa. Science $1985 ; 227$ : 435-438. 
11. Walzer PD, Rutledge ME, Yoneda K, Stahr BJ. Pneumocystis carinii: new separation method from lung tissue. Exp Parasitol 1979; 47: 356-368.

12. Lennette EH (ed.) Manual of clinical microbiology, 3rd edn. Washington, DC, American Society for Microbiology. 1980: 150-159, 533-540, 760-761

13. Maddison SE, Hayes GV, Slemenda SB, Normal LG, Ivey MH. Detection of specific antibody by enzyme-linked immunosorbent assay and antigenemia by counterimmunoelectrophoresis in humans infected with Pneumocystis carinii. $J$ Clin Microbiol 1982; 15: 1036-1043.

14. McNabb SJN, Graves DC, Kosanke SD, Moyer MJ, Ivey MH. Pneumocystis carinii antigen detection in rat serum and lung lavage. J Clin Microbiol 1988; 26: 1763-1771.

15. Raviglione MC. Extrapulmonary pneumocystosis. The first 50 cases. Rev Infect Dis 1990; 12: 1127-1138.

16. Contini C, Romani R, Vullo V, Delia S, Sorice F. Detection of Pneumocystis carinii in urinary samples from a patient with AIDS despite treatment with aerosolized pentamidine. Clin Infect Dis $1994 ; 18$ : 477-478.

17. Sloand E, Laughon B, Armstrong M et al. The challenge of Pneumocystis carinii culture. J Eukaryot Microbiol 1993; 40: $188-195$.

18. Contini C, Manganaro M, Romani $\mathrm{R}$ et al. Activity of terbinafine against Pneumocystis carinii in vitro and its efficacy in the treatment of experimental pneumonia. $J$ Antimicrob Chemother $1994 ; 34: 727-735$.

19. Masur H, Jones TC. The interaction in vitro of Pneumocystis carinii with macrophages and L-cells. J Exp Med 1978; 147: $157-170$.

20. Pottratz ST, Paulsrud J, Smith JS, Martin WJ. Pneumocystis carinii attachment to cultured lung cells by Pneumocystis gp 120, a fibronectin binding protein. J Clin Invest 1991; 88: 403-407.

21. Koziel H, Baik J, Armstrong MJK, Rose RM. Pneumocystis carinii glycoprotein-A is a chemotactic factor for normal human blood monocytes. Am Rev Respir Dis 1993; 147: 33 (abstract).

22. Blumenfeld W, Grifiss JM. In vitro differentiation of humanderived Pneumocystis carinii. J Clin Microbiol 1989; 27 : 480-485.

23. Armstrong MYK, Richards FF. Propagation and purification of rat Pneumocystis carinii in short-term culture. J Protozool 1989; 36: 24S-27S

24. Lee $\mathrm{CH}$, Bauer NL, Shaw MM et al. Proliferation of rat Pneumocystis carinii on cells sheeted on microcarrier beads in spinner flasks. J Clin Microbiol 1993; 31 : 1659-1662.

25. Hong S-T, Steele PE, Cushion MT, Walzer PD, Stringer SL, Stringer JR. Pneumocystis carinii karyotypes. J Clin Microbiol 1990 ; 28: 1785-1795. 